\title{
Bearings Fault Diagnosis based on Wavelet Analysis and Support Vector Machine
}

\author{
Xinli Li \\ School of Control and Computer Engineering \\ North China Electric Power University \\ Baoding, China \\ e-mail: 601279228@qq.com
}

\author{
Wanye Yao \\ School of Control and Computer Engineering \\ North China Electric Power University \\ Baoding, China \\ e-mail: yaowanye@163.com
}

\author{
Xiao Yang \\ School of Energy Power and Mechanical Engineering \\ North China Electric Power University \\ Baoding, China \\ e-mail: 350964520@qq.com \\ Jianming Wang \\ join Bright digital power technology Co, Ltd \\ Beijing, China \\ e-mail:wangjianming010@126.com
}

\begin{abstract}
Drive system of generator is an important part of wind turbine, once the fault occurs it will cause huge economic losses. For this reason, in this paper, a fault diagnosis method is proposed for generator rolling bearings, it combined with wavelet packet and support vector machine. Acquisition of wind farm FAG6332 rolling bearings vibration signal in real-time database, which contains the normal operation 、 inner ring fault and outer ring fault. Firstly, using wavelet packets decomposed the vibration signals into different frequency bands, so extract energy spectrum as a fault feature vector, then put the fault feature vector into the support vector machine training the SVM; Using MATLAB for simulation experimental, put the test sample into the trained SVM for fault pattern recognition, the results show that the fault diagnosis model can make a $\mathbf{9 3 . 3 3 3 3 \%}$ high precision, can make a fast and effective fault diagnosis for rolling bearings. The conclusion is that the fault diagnosis method can recognition the drive system fault, improve the utilization of wind turbines, and it offer a
\end{abstract} good guide to the operation of wind farm.

Keywords: Support vector machine; Rolling bearings; Fault diagnosis; Wavelet packet; Wind Turbines

\section{INTRODUCTION}

Rolling bearings of generator is one of the most important parts of rotating machinery, its operating status can directly affect the performance of wind turbine ${ }^{[1]}$. So how to quickly and accurately find the fault reason is the urgent demand of the current industrial development. When the rolling bearing failure, its vibration acceleration will changed, then decomposed the vibration signal, using wavelet analysis to extract energy value of each band, which is one of the most commonly used method [2]. However, the traditional wavelet analysis can not clearly distinguish the frequency of the high frequency portion, however, when the bearing fault, its vibration signal decomposition is very important in high frequency, therefore we proposed wavelet packet fault feature extraction method, which for low and high frequency portion of the signal can be fine decomposition; wavelet packet decomposition can effectively reflect the timefrequency characteristics of vibration signal, for the nonstationary and transient signals can be accurate extracted the fault feature ${ }^{[3]}$.After accurately extract the fault feature we should to choose an efficient fault diagnosis method. Traditional fault diagnosis require accurate mathematical model, which can not be established in most of complex nonlinear systems ${ }^{[4]}$,and the artificial neural network exist the problems of complex structure, too learning or less learning and local minima ${ }^{[5]}$. Based on the above problems a fault diagnosis method based on support vector machine is proposed, which find the best compromise between learning accuracy of specific training samples and learning ability of correctly identify any learning samples, in order to obtain the best classification results. SVM solve the problem of complex nonlinear systems sample dimension disaster and the local minimum; compared with other fault diagnosis methods, it has greater versatility, effectiveness and generalization ability, it has been widely used in classification problem of fault diagnosis ${ }^{[6]}$.

\section{WAVELET PACKET ANALYSIS}

Wavelet packet method is based on an orthogonal decomposition of multi-resolution analysis, which put the original signal by any time-frequency resolution decomposition, then its corresponding time-frequency component is projected onto the wavelet space of different frequency bands, each band signal energy spectrum contains detailed information of the original signal, which can extract the signals eigenvectors in different frequency bands ${ }^{[7]}$.

The step of wavelet packet decomposition extract fault features ${ }^{[8]}$

1)For the vibration signal wavelet packet decomposition

The vibration signal of rolling bearings for 3 layer wavelet decomposition, the structure shown in Fig.1, extraction layer 3 from low to high frequency coefficients of wavelet packet : $\left(x_{3}^{0}, x_{3}^{1}, x_{3}^{2}, x_{3}^{3}, x_{3}^{4}, x_{3}^{5}, x_{3}^{6}, x_{3}^{7}\right)$ 


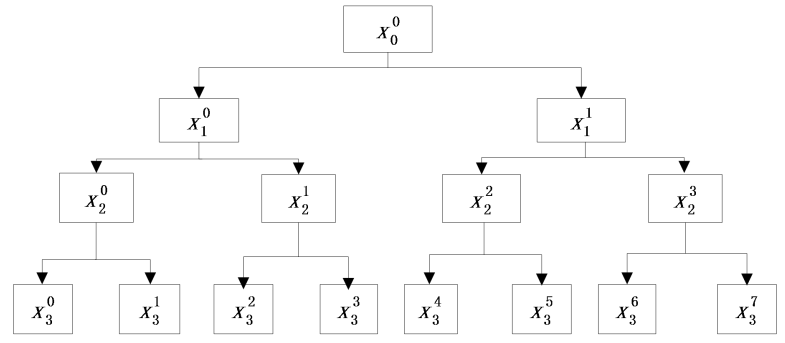

Figure 1. Structure of wavelet packet decomposition

\section{2)Wavelet packet decomposition coefficients} reconstruction

The reconstructed signal by denoted $S_{3 i}(i=0,1 \ldots 7)$, then analysis the nodes of the third layer, the total signal $\mathrm{S}$ is:

$S=S_{30}+S_{31}+S_{32}+S_{33}+S_{34}+S_{35}+S_{36}+S_{37}$

3) The total energy of each band signal

Reconstructed signal corresponding to the energy of $E_{3 i}=\int\left|S_{i k}\right|^{2} d t=\left.\sum_{i=1}^{n} x_{i k}\right|^{2}, i=1,2 \ldots . \mathrm{n}$, where the $x_{i k}$ represents the reconstructed signal S amplitude of discrete points .

4) Extract features vector

According to the vibration characteristic, the features vector is : $T=\left[E_{30}, E_{31}, E_{32}, E_{33}, E_{34}, E_{35}, E_{36}, E_{37}\right]$.

\section{5) Normalization}

The energy value of each band signals are usually larger, it will increase the complexity of the later data analysis, so need the normalization process. The total energy of the signal is $E=\sum_{i=0}^{2^{i}-1} E_{i k}$, after normalized the feature vector as follows:

$T^{*}=\left[E_{30}, E_{31}, E_{32}, E_{33}, E_{34}, E_{35}, E_{36}, E_{37}\right] / E$.

Select a large number of bearing vibration date about normal and fault signals, repeat the above steps, we can get the samples of feature vector fault.

\section{SUPPORT VECTOR MACHINE THEORY}

The main idea of SVM is: by a linear transformation the input vector are mapped to the high dimensional feature space, then establishment of a hyper plane as a decision plane in the feature space, make the edge of the spacer between the positive and negative examples of that plane to maximize ${ }^{[9]}$.

1) Linearly separable establish optimal hyper plane ${ }^{[10]}$ :

Training sample $\left\{x_{i}, y_{i}\right\}_{i=1}^{M}$, which $x_{i}$ is the i-th input mode samples, $y_{i}$ output the type of fault (expected response), the hyper plane satisfy the equation is:

$y_{i}\left(\omega x_{i}+b\right)-1 \geq 0$; where $\omega$ is an adjustable weight vector that is the normal vectors of hyper plane, $b$ on behalf of bias, it is a constant factor.

Hyper plane problem turn into solving quadratic optimal programming problems (1):

$$
\left\{\begin{array}{l}
\min \frac{\|\omega\|^{2}}{2} \\
\text { s.t } \quad y_{i}\left(\omega x_{i}+b\right)-1 \geq 0
\end{array}\right.
$$

Introduction of Lagrange multipliers to get an optimal value (2):

$$
\left\{\begin{array}{l}
\omega_{1}=\sum_{i=1}^{N} a_{i} y_{i} x_{i} \\
b_{1}=1-\omega_{1} x^{(s)}
\end{array}\right.
$$

Which $a^{*}{ }_{i}$ is the optimal Lagrange multiplier, $\omega_{1}, b_{1}$ is the most optimal value for hyper plane. Based on the above theory, for the test data, use judgment function (3) which can be obtained classification result

$$
\operatorname{sgn}\left(\sum_{i=1}^{N} a_{i}^{*} y_{i} x_{i} x+b\right)
$$

\section{2)Linearly inseparable establish optimal hyper plane ${ }^{[11]}$ :}

By a linear transformation, input $x$ through nonlinear mapping to a high dimensional feature space $\phi(x)$, then can be achieved linearly separable pattern hyper plane establish in high-dimensional space; however this method need to introduce penalty coefficient $\mathrm{C}$ and non-negative slack factor $\xi_{i}$, the hyper plane problem will be transformed into (4):

$$
\left\{\begin{array}{l}
\min \frac{\|\omega\|^{2}}{2}+C \sum_{i=1}^{M} \xi_{i} \\
\text { s.t } \quad y_{i}\left(\omega^{T} \phi\left(x_{i}\right)+b\right)-1+\xi_{i} \geq 0
\end{array}\right.
$$

Based RBF kernel function(5)

$$
K\left(x_{i}, x_{j}\right)=\phi\left(x_{i}\right)^{T} \phi\left(x_{j}\right)=\exp \left(-\gamma\left\|\phi\left(x_{i}\right)-\phi\left(x_{j}\right)\right\|^{2}\right)(5)
$$

According to (6) get the result classification:

$$
f(\phi(x))=\operatorname{sgn}\left(\sum_{i=1}^{m} a^{*}{ }_{i} y_{i} K\left(\phi\left(x_{i}\right), x\right)+b\right)
$$

\section{BEARINGS FAULT DIAGNOSIS}

The basic idea of rolling bearings fault diagnosis based on wavelet packet analysis and support vector machine is: firstly, use wavelet packet algorithm for vibration signal 3 layer decomposition, make the vibration signal is decomposed into separate bands, each band reconstructed signal, and the signal energy in different frequency bands within the change reflects bearing different operating states, it can be extracted wavelet packet energy as a feature vector; put the extracted feature vectors into the support vector machine, to SVM classifier training, after the training is completed, put the test sample into the already trained SVM for fault type identification. The fault diagnosis flowchart shown in Fig. 2:

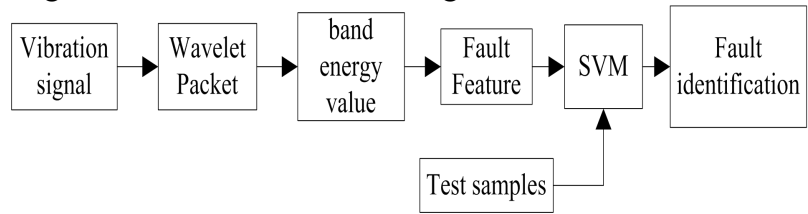

Figure 2.Fault diagnosis process 
Experimental data from a wind power plant generator bearing on the historical database of 105 data, the sampling frequency is $2500 \mathrm{HZ}$. The data includes bearing normal, bearing outer ring fault and bearing inner ring fault respectively 35 . Rolling bearings energy spectrum of three states as shown in Fig.3. From the Fig.3, we can see that bearing in different states which the energy spectrum corresponding to the shape of the bar chart is not the same, So it can extract different energy bands characteristic value as the failure vectors.

normal outer ring fault inner ring fault

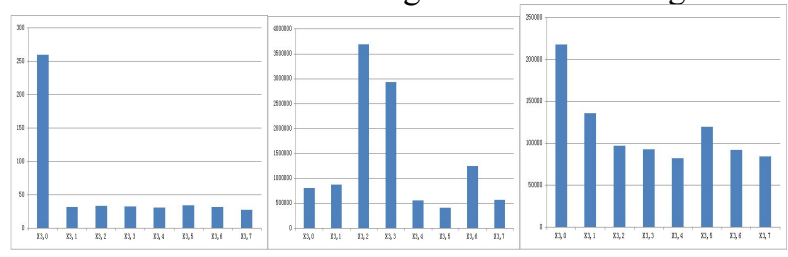

Figure 3.Bearing three states energy comparison chart

Bearing normal, inner ring fault and outer ring fault timedomain characteristic shown in Fig 4.Bearing vibration signal 3 layers wavelet packet decomposition use: $\mathrm{x}=\operatorname{wpdec}\left(\mathrm{s}, 3, \mathrm{db}^{\prime} 0^{\prime}\right)$, from the decomposition result select 3 layer from low frequency to high frequency components. as shown, Fig.5 is the bearing normal wavelet packet Coefficients, Fig. 6 is the bearing inner fault Wavelet Packet Coefficients, Fig.7 is the bearing outer fault Wavelet Packet Coefficients.
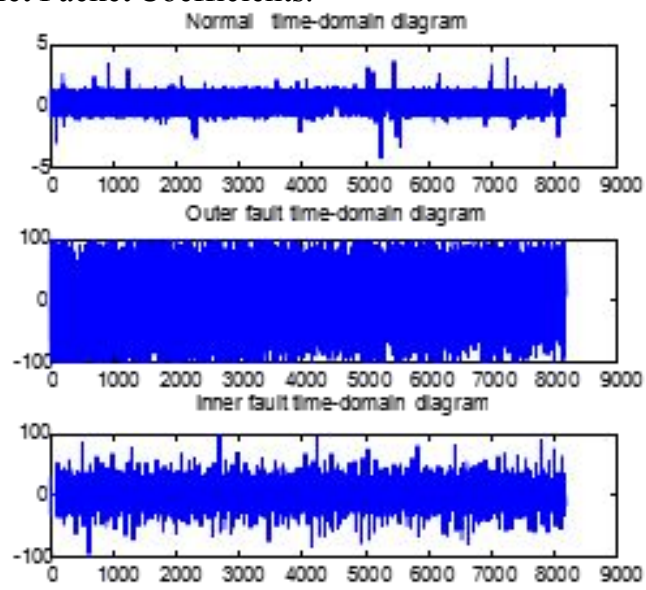

Figure 4.time-domain diagram of bearing
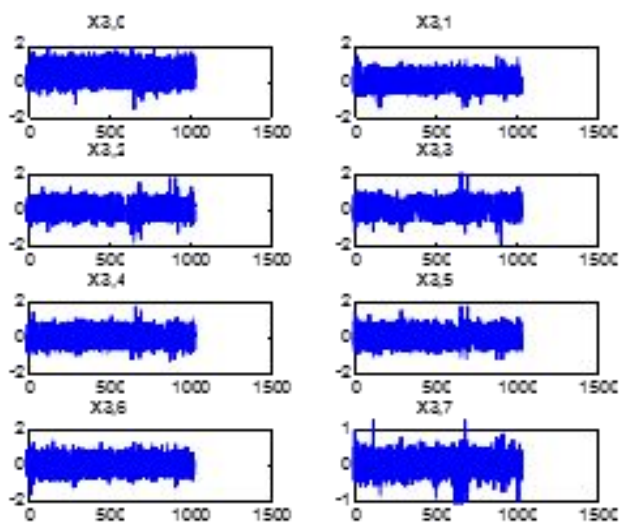

Figure 5.Bearing normal wavelet packet Coefficients
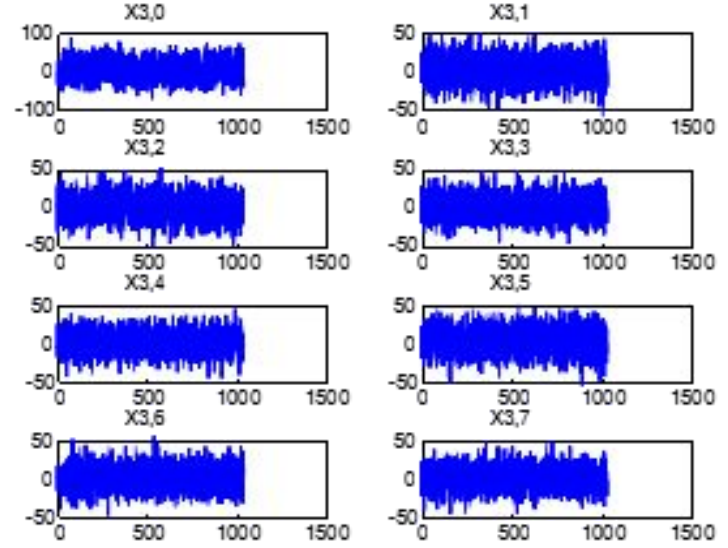

Figure 6.Bearing inner fault Wavelet Packet Coefficients
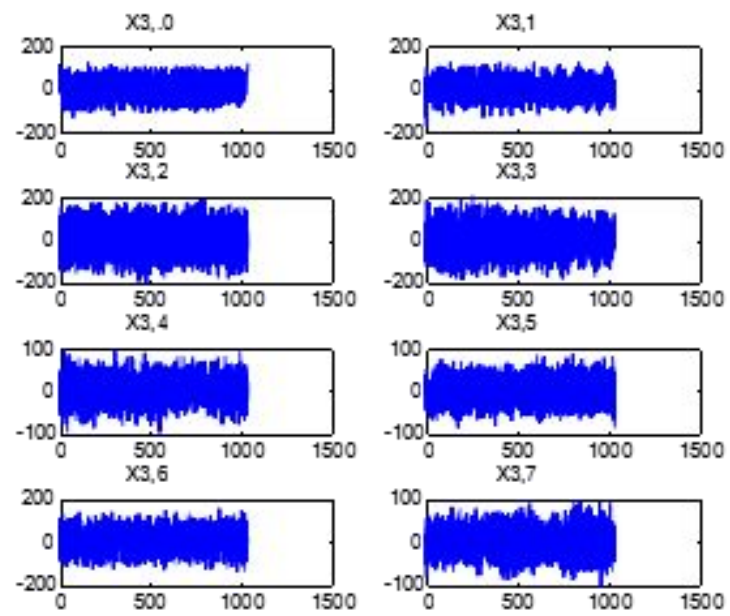

Figure 7.Bearing outer fault Wavelet Packet Coefficients

The reconstructed signal is $S_{3 i}$, then calculate the total energy of each band signal, $E(i)=$ norm $(\operatorname{wpcoef}(t,[n, i-$ $1]), 2) *$ norm (wpcoef (t, [n, i-1]), 2);After one of the energy-normalized the feature vector obtained as follows: $T^{*}=\left[E_{30}, E_{31}, E_{32}, E_{33}, E_{34}, E_{35}, E_{36}, E_{37}\right] / E$.

\section{B. Establish fault diagnosis model}

1) parameter settings

SVM classifier using one-to-one method to create multiple classifiers, the number of classifiers is $n(n-1) / 2$, $\mathrm{n}$ is the number of categories fault. In this paper, the states is normal, inner ring fault and outer ring fault, so requiring three SVM, setting the normal operation as 1 , failure of the bearing inner ring 2 , the bearing outer ring fault is 3 , the output vector $[1,2,3]$. Form the 105 data, choose 90 groups of data as training samples, 15 groups as test data.

2)Model building

model=svmtrain(train_label, train, '-c 2 -g 1'); Where the penalty parameter-c and function arguments $-\mathrm{g}$ is the best parameter through CA methods adopted.

3)Predicted output

[predict_label,accuracy]=svmpredict(test_label,test,model);Its output representative of accuracy and category labels of test samples, forecast classification results shown in Table 2,Accuracy $=93.3333 \%(14 / 15)$. 
TABLE.I Test Classification Results

\begin{tabular}{|c|c|c|c|c|c|c|c|c|c|c|}
\hline \multirow[b]{2}{*}{ Samples } & \multicolumn{8}{|c|}{ Characteristic value } & \multirow{2}{*}{$\begin{array}{l}\text { Predict } \\
\text { result }\end{array}$} & \multirow{2}{*}{$\begin{array}{l}\text { Actual } \\
\text { result }\end{array}$} \\
\hline & $T_{0}^{*}$ & $T_{1}^{*}$ & $T_{2}^{*}$ & $T_{3}^{*}$ & $T_{4}^{*}$ & $T_{5}^{*}$ & $T_{6}^{*}$ & $T_{7}^{*}$ & & \\
\hline 1 & 1 & 0 & 0.003 & 0.006 & 0.001 & 0.010 & 0.008 & 0.004 & 1 & 1 \\
\hline 2 & 1 & $7.2 \mathrm{e}^{-04}$ & 0 & 0.003 & 0.002 & 0.004 & 0.007 & 0.001 & 1 & 1 \\
\hline 3 & 1 & 0.003 & 0.003 & 0.003 & 0 & 0.003 & 0.01 & 0.002 & 1 & 1 \\
\hline 4 & 1 & 0.633 & 0.040 & 0.824 & 0.362 & 0.458 & 0.274 & 0 & 3 & 1 \\
\hline 5 & 1 & 0.006 & 0.006 & 0.003 & 0.00 & 0.005 & 0.007 & 0 & 1 & 1 \\
\hline 6 & 0.835 & 0.748 & 0.642 & 0.821 & 1 & 0.783 & 0.269 & 0 & 2 & 2 \\
\hline 7 & 0.839 & 0.804 & 0.467 & 0.476 & 0.792 & 1 & 0.269 & 0 & 2 & 2 \\
\hline 8 & 1 & 0.850 & 0.765 & 0.359 & 0.072 & 0.284 & 0.198 & 0 & 2 & 2 \\
\hline 9 & 0.999 & 1 & 0.419 & 0.547 & 0.641 & 0.836 & 0.261 & 0 & 2 & 2 \\
\hline 10 & 1 & 0.902 & 0.477 & 0.559 & 0.678 & 0.748 & 0.429 & 0 & 2 & 2 \\
\hline 11 & 0.12 & 0.398 & 0.461 & 1 & 0.462 & 0.153 & 0.024 & 0 & 3 & 3 \\
\hline 12 & 1 & 0.414 & 0.039 & 0.147 & 0 & 0.006 & 0.208 & 0.079 & 3 & 3 \\
\hline 13 & 1 & 0.404 & 0 & 0.204 & 0.030 & 0.166 & 0.189 & 0.261 & 3 & 3 \\
\hline 14 & 0 & 0.471 & 0.599 & 0.628 & 1 & 0.057 & 0.472 & 0.510 & 3 & 3 \\
\hline 15 & 0 & 0.498 & 0.399 & 0.993 & 1 & 0.146 & 0.545 & 0.927 & 3 & 3 \\
\hline
\end{tabular}

Test set prediction classification and the actual classification compared results are shown in Fig.8:

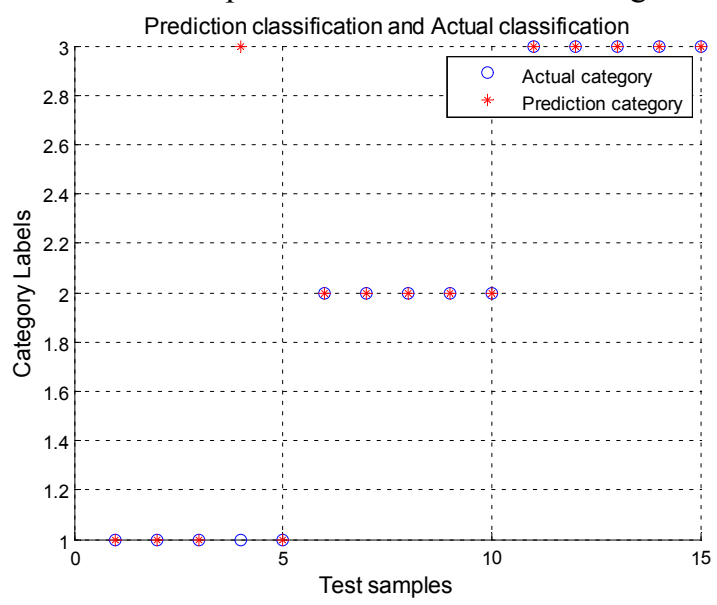

Figure.8

Result analysis:

Form the table 2 and Fig. 8 we can see that, the 4th sample of bearing normal state mistakenly identified as the bearing outer ring fault, the other samples predictive value consistent with the actual value ,the correct rate reached $93.3333 \%$. The test results demonstrated the high accuracy of fault diagnosis model based on support vector machines.

\section{ACKNOWLEDGMENT}

Form the above experiment results we can get the conclusion that, the rolling bearings fault diagnosis combined with wavelet packet decomposition and support vector machine can achieve accurately fault feature vectors extract and effectively classification of rolling bearings normal, inner fault and outer fault; wavelet packet decomposition suited to handle non-stationary signals, can accurately and effectively extract fault feature vector; Using MATLAB simulation, put the test samples into the trained support vector machine, the classification accuracy reached $93.3333 \%$, within the error allowable range, we can consider that the model is accurately. Fault diagnosis based on wavelet packet decomposition and support vector machine, can effectively improved the fault diagnosis speediness, effectiveness and accuracy.

\section{REFERENCES}

[1] Yang Min-min. Based on Support Vector Machines of Bearing Fault Diagnosis Method Study[D]. Jiangxi University,2012

[2] Wang Xian-feng,Li Hong-tao, Oberhausen noise of Nondestructive Testing Technology, Bearing. 8 (2003) 27-28.

[3] Rubini R, Meneghetti U. Application of the envelope and wavelet transform analyses for the diagnosis of incipient faults in ball bearings[J]. Mechanical systems and signal processing, 2001, 15(2): 287-302.

[4] Gui Wei-hua, Liu Xiao-ying. Based on the complex process of artificial intelligence algorithms fault diagnosis[J].Control Engineering,2002,04:1-6.

[5] Qi Heng-nian. Review of Support Vector Machine and Its Applications[J].Computer Engineering.2004,30(10):6-9.

[6] Peng W, Luo X. Research on vibrant fault diagnosis of hydroturbine generating unit based on wavelet packet analysis and support vector machine[J]. Proceeding of the CSEE, 2006, 26(24): 164-167.

[7] Huang Jian-hong, Jiang Qing-nian and Zhang Xun-hua, Wavelet Envelope Analysis Based on Wavelet Packet Energy Feature and Its Application in Failure Diagnosis of Rolling Bearing, Journal of Nanchang University: (Natural Science). 30 (2006) 402-405.

[8] Zhou Wei, Wavelet Analysis based on MATLAB, Xi'an University of Electronic Science and Technology Press, Xi'an, 2010. pp. 21-30.

[9] ZHANG L, HANG B. Relationship between supper vector set and kernel functions based SVM and three-layer feed-forward networks[J]. Chinese J of Computer, 2002, 25(7): 1-5.

[10] Vapnik V N, Vapnik V. Statistical learning theory[M]. New York: Wiley, 1998.

[11] Burges C J C. A tutorial on support vector machines for pattern recognition[J]. Data mining and knowledge discovery, 1998, 2(2): 121-167. 